\title{
Colloidal Quasi-One-Dimensional Dual Semiconductor Core/Shell Nanorod Couple Heterostructures with Blue Fluorescence
}

Received 00th January 20xx, Accepted 00th January 20xx

DOI: $10.1039 / x 0 x \times 00000 x$
Dechao Chen, ${ }^{a}$ Aixiang Wang, ${ }^{b}$ Hongbo Li, $^{c}$ Laura Abad Galán, ${ }^{a}$ Cong Su, ${ }^{d}$ Zongyou Yin, ${ }^{e}$ Massimiliano Massi, ${ }^{a}$ Alexandra Suvorova, ${ }^{f}$ Martin Saunders, ${ }^{f} \mathrm{Ju} \mathrm{Li}^{\mathrm{d}}{ }^{\mathrm{d}}$ Amit Sitt, ${ }^{* \mathrm{~g}}$ and Guohua Jia*a

\begin{abstract}
Herein we report a nanorod couple heterostructure made of dual semiconductors, in which two parallel aligned ZnSe nanorods are connected by the growth of ZnS on both end and side facets, producing hetero-ZnS (short arms)-ZnSe (long arms)/ZnS shell nanorod couples. As evidenced by electronic structure studies, both experimental and theoretical, such core/shell nanorod couple heterostructures can act as a platform to precisely tailor the quantum confinement of charge carriers between the constituting components within a single nano-object, generating blue fluorescence after the overgrowth of an alloyed $\mathrm{ZnCdS}$ layer on the heterostructures. We foresee the mechanistic insights gained and electronic structures revealed in this work would shed light on the rational design of more complex heterostructures with novel functionalities.
\end{abstract}

\section{Introduction}

Nano-heterostructures that integrate different components together to provide a system with unique dimensionality-dependent integrative and synergic effects ${ }^{1-6}$ are of significant importance due to their diverse applications in solar energy harvesting, ${ }^{7,8}$ catalysis, ${ }^{9,10}$ electronics, ${ }^{11,12}$ biological imaging, ${ }^{13,14}$ and medical diagnostics and treatment. ${ }^{15,16}$ The recent advancements in the protocols of the colloidal synthesis enable the construction of multicomponent structures with increasing chemical complexity that can be achieved in a predictable manner via a series of sequential synthetic procedures. ${ }^{1,2,12,17,18}$ In most cases, the preparation of heterostructures involves the sequential growth of one material onto another. ${ }^{1,2,18}$ However, heterostructures that are formed by the joining of two nanocomponents of the same composition together using a second material are less common in the literature.

As a benchmark heterostructure, CdSe seeded CdS core/shell quantum rods with green to red fluorescence have received significant attention because it provides a platform for the stimulating studies of the elongated nanocrystals in a

a. Curtin Institute of Functional Molecules and Interfaces, School of Molecular and Life Sciences, Curtin University, GPO Box U1987, WA 6845, Australia E-mail: guohua.jia@curtin.edu.au

b. School of Chemistry and Chemical Engineering, Linyi University, Linyi 276005, China

School of Materials Science and Engineering, Beijing Institute of Technology, Haidian District, Beijing 100081, China.

${ }^{d .}$ Department of Nuclear Science and Engineering and Department of Materials Science and Engineering, Massachusetts Institute of Technology, Cambridge, MA 02139, USA

${ }^{e}$ Research School of Chemistry, The Australian National University, Canberra, Australian Capital Territory 2601, Australia

f. Centre for Microscopy, Characterization and Analysis (CMCA) and School of Molecular Sciences, The University of Western Australia Perth, WA 6009, Australia

g. Raymond and Beverly Sackler Faculty of Exact Sciences, School of Chemistry Te Aviv University, Tel Aviv 6997801, Israel E-mail:amitsitt@tauex.tau.ac.il

† Footnotes relating to the title and/or authors should appear here.

Electronic Supplementary Information (ESI) available: [details of any supplementary information available should be included here]. See DOI: $10.1039 / x 0 x x 00000 x$ variety of applications in assemblies, photocatalysis, optoelectronic devices, and bio-labeling. ${ }^{19-23}$ However, due to the small band gap of the core material of CdSe and the band offsets of the CdSe/CdS core/shell structure, it is difficult to tune the fluorescence of the $\mathrm{CdSe} / \mathrm{CdS}$ core/shell quantum rod heterostructures into the blue spectral range. Manna and coworkers have developed a sequential cation exchange approach, in which the $\mathrm{Cd}^{2+}$ ion is replaced by $\mathrm{Cu}^{+}$and then by $\mathrm{Zn}^{2+}$, to prepare blue-emitting $\mathrm{ZnSe/ZnS} \mathrm{core/shell} \mathrm{quantum}$ rods, ${ }^{24}$ but this exchange is formed post-synthesis and involves multi-steps. Hence, to date, the straightforward synthesis of semiconductor quantum rods with blue fluorescence remains highly challenging.

Herein, we report a wet-chemical method for fabricating hetero- ZnS (short arm)-ZnSe/ZnS core/shell (long arm) nanorod couples that are formed by bridging precisely two parallelly aligned ZnSe nanorods with $\mathrm{ZnS}$ at their edges. Mechanistic studies reveal that the formation of this heterostructure is attributed to the growth of $\mathrm{ZnS}$ between the end facets of each two parallelly aligned ZnSe nanorods, constituting ZnS (short arms)-ZnSe/ZnS core/shell (long arms) nanorod couples. Effective mass approximation (EMA) simulations reveal that the electron occupies only ZnSe states, while the hole occupies a ZnS-ZnS state. Over-coating the nanorod couples with a layer of CdS produces $\mathrm{ZnS}-\mathrm{ZnSe} / \mathrm{ZnCdS}$ core/shell nanorod couples with blue fluorescent, in which the alloyed ZnCdS layer eliminates the surface traps and improves the quantum efficiency. The heterostructures were further converted into hetero-PbS (short arms)-PbSe (long arms)/PbS nanorod couples through a cation exchange reaction.

\section{Results and discussion}

Controlled Synthesis and growth mechanism of hetero- ZnS (short arm)-ZnSe/ZnS core/shell (long arm) nanorod couples

The hierarchical self-assembly processes in the growth of nanorod couples allow the separation of the intermediate products from the reaction system and the manipulation of the particle growth in a controlled manner. ${ }^{25,26}$ We took this 


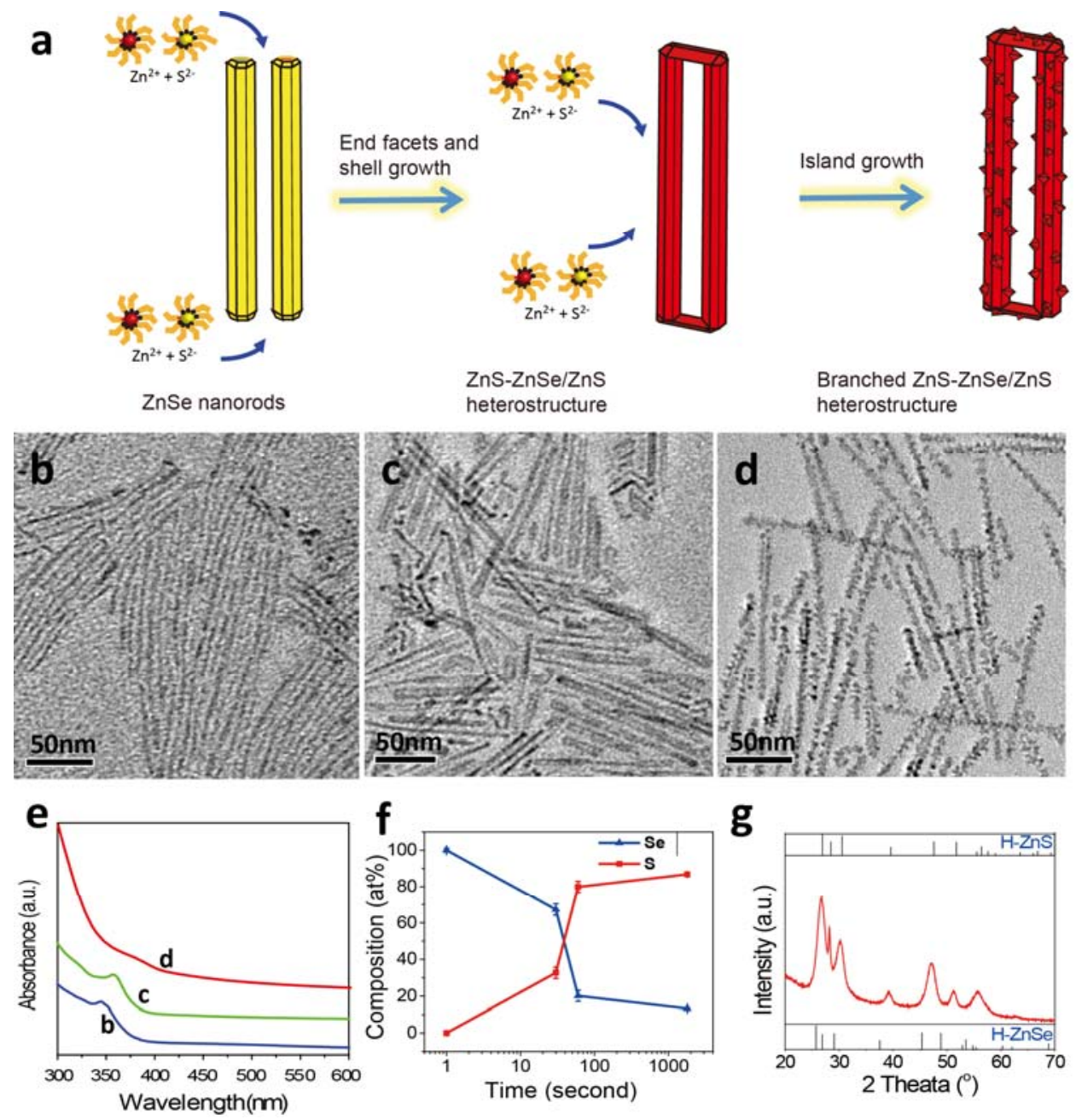

Fig. 1 (a) A schematic illustration showing a controlled synthesis of ZnS (short arms)-ZnSe/ZnS core/shell (long arms) heterostructures. (b-d) TEM images of (b) the original thin ZnSe nanorods, (c) the hetero-ZnS-ZnSe/ZnS core/shell nanorod couples obtained after 30 seconds at $280{ }^{\circ} \mathrm{C}$, and (d) the branched hetero- ZnS-ZnSe/ZnS core/shell nanorod couples obtained after 30 minutes at $280{ }^{\circ} \mathrm{C}$. (e) Absorption spectra corresponding to (b-d). (f) Chemical composition of Se (\%) and S (\%) of branched hetero-ZnS-ZnSe/ZnS core/shell nanorod couples as a function of the reaction time (1 second, 30 seconds, 1 minute and 30 minutes, respectively). (g) XRD patterns of branched hetero- ZnS-ZnSe/ZnS core/shell nanorod couples.

into advantage by incorporating both $\mathrm{Zn}$ and $\mathrm{S}$ precursors together with the purified ZnSe nanorods into the reaction system to synthesize hetero-ZnS (short arm)-ZnSe/ZnS core/shell (long arm) nanorod couples (Figure 1a). Surprisingly, after the reaction has evolved for 30 seconds, $\mathrm{Zn}$ (zinc nitride) and $S$ precursors (1-dodecanthiol) integrated two ZnSe nanorods into a single hetero-nanorod couple (Figure $1 \mathrm{~b}$ ) by the growth of $\mathrm{ZnS}$ on both end facets of two parallelly aligned ZnSe nanorods (Figure 1c). Further growth of ZnS islands on side facet of the long arms was obtained as the reaction continued (Figure 1d). Analogous to the previously reported single-component $\mathrm{ZnSe}$ nanorods couples, ${ }^{26}$ the driving forces responsible for the initial pairing of ZnSe nanorods with ZnS are dipole-dipole and crystal-crystal interactions. The pairing of $\mathrm{ZnSe}$ rods via bridging both end facets by $\mathrm{ZnS}$ is formed via a self-limited assembly mechanism, because once the end facets of ZnSe are blocked by the growth ZnS, the hetero-ZnSe-ZnS nanorod couple cannot interact with another ZnSe nanorod. ${ }^{26}$
Upon the growth of ZnS shell, the absorption onset exhibits a slight red-shift of $7 \mathrm{~nm}$ as a result of the leakage of the excitonic wave function into the $\mathrm{ZnS}$ layer. ${ }^{27,28}$ A prolonged reaction time of 30 minutes (Figure $1 \mathrm{~d}$ ) produced branched hetero-ZnS-ZnSe/ZnS nanorod couples with ZnS decorating the surface of the nanorod couple heterostructures. The corresponding absorption onset is slightly smeared, but clearly, a further red shift is observed. The islands growth of $\mathrm{ZnS}$ at prolonged time may be a result of the lattice strain existing between interfaces of the $\mathrm{ZnSe}$ and $\mathrm{ZnS},{ }^{29}$ which may also result in the red shift of absorption as previously observed in $\mathrm{ZnSe} / \mathrm{ZnS}$ core/shell nanoparticles. ${ }^{30}$ Statistical analysis (Figure S1) shows that the lengths of the original nanorods (Figure $1 \mathrm{~b}$ ), intermediate (Figure $1 \mathrm{c}$ ) and final products (Figure 1d) are almost constant ( $72.0 \mathrm{~nm}$ to $78.7 \mathrm{~nm})$, but their widths change from $2.5 \mathrm{~nm}$ to $5.6 \mathrm{~nm}$, and then to $7.5 \mathrm{~nm}$. The increase of width correlates to both the growth of $\mathrm{ZnS}$ on both end and side facets of two parallelly aligned ZnSe nanorods. A 


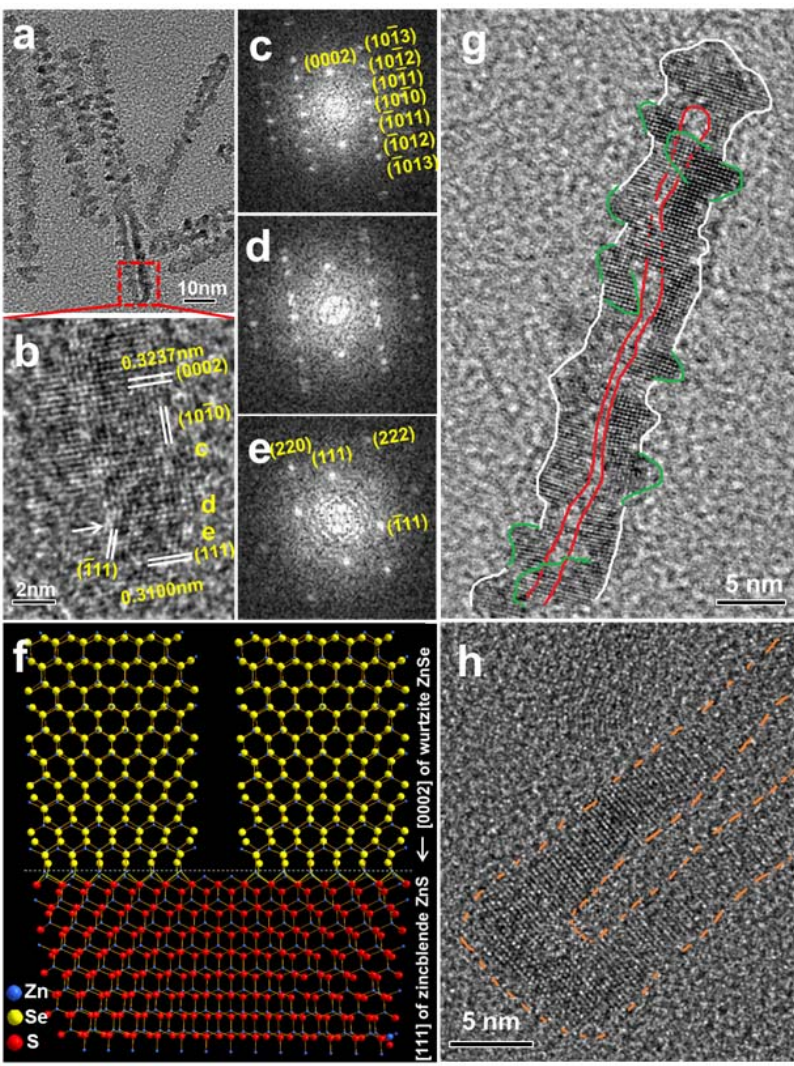

Fig. 2 (a) TEM image. (b) HRTEM image of the short arm marked by dashed rectangle in (a). The boundary between wurtzite hexagonal ( $\mathrm{H}-$ ) $\mathrm{ZnSe}$ and zincblende cubic (C-) $\mathrm{ZnS}$ is labelled by an arrow. (c-e) FFT of selected areas in (b) revealing the crystallographic relations. The diffraction patterns in (c) and (e) match $\mathrm{H}-\mathrm{ZnSe}$ and $\mathrm{C}-\mathrm{ZnS}$, respectively. (f) Schematic atomic crystal structure corresponding to the region depicted in (b). (g) HRTEM image of an individual branched nanorod couple. White lines outline boundary of the branched nanorod couple, green lines outline the ZnS branches, and red lines outline the gap between two rods. (h) HRTEM image of the branched hetero- ZnS-ZnSe/ZnS/CdS core/shell/shell nanorod couples revealing a clear gap (marked by dashed orange lines) between two rods.

distinct spacing between two rod components (Figure 1c, 1d) is seen, which can be attributed to the steric hindrance of the oleylamine ligands passivating on the surface of each rod components. Energy dispersive X-ray (EDX) spectroscopy analysis shows that the atomic ratio of Se:S decreases as a function of the reaction time (Figure 1f), which is consistent with the growth of ZnS on the ZnSe nanorod components. The powder X-ray diffraction (XRD) patterns of hetero-ZnSZnSe/ZnS core/shell nanorod couples present a shift to higher angles with respect to those of pure $\mathrm{ZnSe}$, which further confirms the growth of the ZnS (Figure 1g). Using bis(trimethylsilyl)sulfide as the sulfur source instead of 1dodecanethiol while keeping other synthetic conditions constant also produced hetero-ZnS-ZnSe/ZnS core/shell

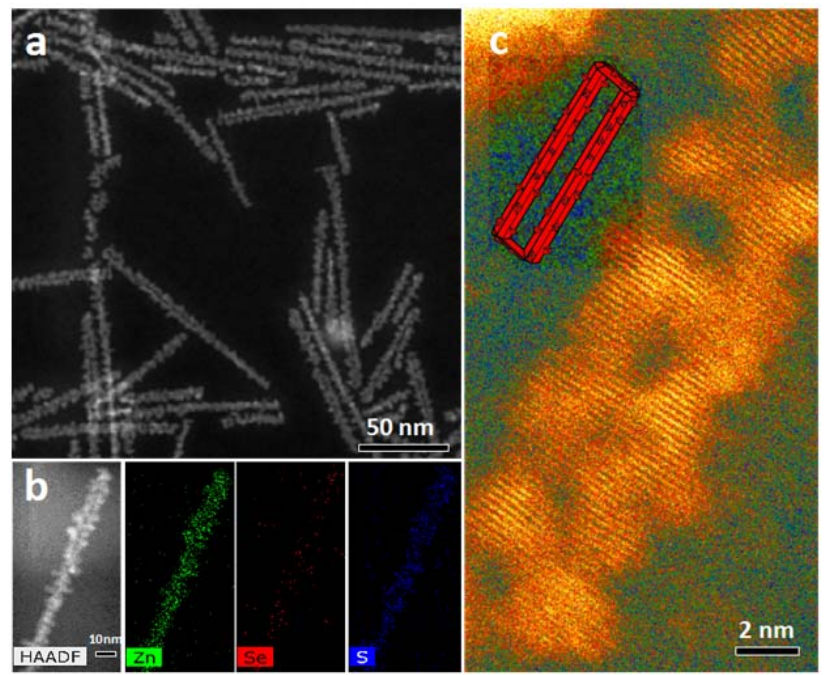

Fig. 3 (a) HAADF-STEM image of hetero-ZnS-ZnSe/ZnS core/shell nanorod couples showing a spacing between each two rods. (b) HAADF-STEM image and elemental maps of an individual hetero- ZnS-ZnSe/ZnS core/shell nanorod couple. (c) A high-resolution Z-contrast STEM image of an individual hetero- ZnS-ZnSe/ZnS core/shell nanorod couple.

nanorod couples (Figure S2) with an even smaller spacing between the two ZnSe rods. The high activity of bistrimethylsilyl)sulfide leads to the fast growth of $\mathrm{ZnS}$ and its high etching capability at elevated temperatures may result such a small spacing between each rod components of the hetero- nanorod couples. ${ }^{31}$ This demonstrated the generality of this synthetic approach for compositing hetero- nanorod couples. The above control experiment also demonstrated that two elongated nanorods can be integrated into a single nanoobject in a predefined way through material engineering and optimization of synthetic protocols.

We used transmission electron microscope (TEM) imaging in combination with fast Fourier transform (FFT) analysis to reveal how hetero- ZnS-ZnSe/ZnS core/shell nanorod couples form from two parallelly aligned $Z n S e$ nanorods in the presence of $\mathrm{Zn}$ and S precursors. Figures S2a and S3 show the branched hetero- ZnS-ZnSe/ZnS core/shell nanorod couples with different dimensions. Close inspection on the connecting areas of the heterostructures (labelled by dashed red rectangle in Figures 2a, S4a and S4f) show that a single heterostructure is formed through $\mathrm{ZnS}$ growth on both end facets of two parallelly aligned ZnSe nanorods. The (111) plane of zincblende ZnS grows on the (0002) plane of wurtzite ZnSe nanorods, creating the connecting areas (Figures $2 b, 54 b$ and $54 g$ ), as confirmed by the presence of two sets of diffraction patterns (Figures $2 \mathrm{~d}$, S4d and S4i) of both zincblende ZnS (Figures 2e, S4e and S4j) and wurtzite ZnSe (Figures 2c, S4c and S4h) in close proximity of the boundary. We attribute the growth of (111) zincblende $\mathrm{ZnS}$ on the (0002) plane of the ZnSe wurtzite to the moderate lattice mismatch between these two planes, which is about $3.9 \%$ (Table S1). ${ }^{32-34}$ Based on above diffraction analysis, we propose the atomistic crystal structure shown in Figure $2 f$ to explain how zincblende $\mathrm{ZnS}$ short arms join the 


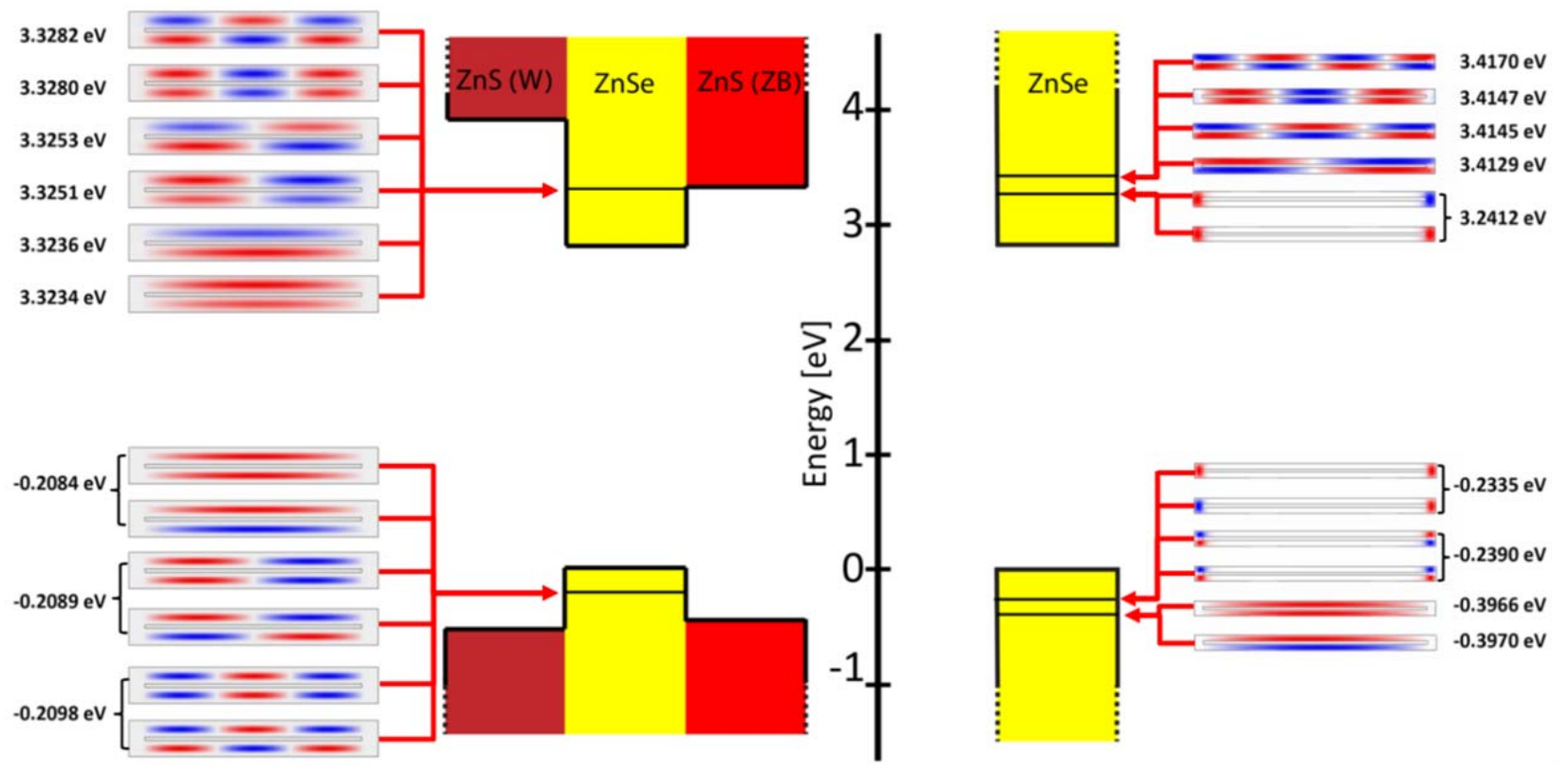

Fig. 4 Electronic structures of hetero- ZnS-ZnSe nanorod couples (left panel) compared with homo-ZnSe nanorod couples (right panel). A Zoom-ins on the near band-edge energy levels and envelope wave functions of electron and hole states are depicted in top and bottom panels, respectively. The wave functions are portrayed at the cross-section along the $z x$ plane $(y=0)$. White is 0 , and red and blue are the + and - phases, respectively. Electron and hole states are presented in different energy scales for clarity.

end facets of two parallelly aligned ZnSe nanorods.

The orientation of the crystal lattices of branched structures is similar to that of the ZnSe rod couples, as is confirmed by HRTEM characterization (Figures $2 \mathrm{~g}$, S5) and the FFT analyses (insets of Figure S5). An evident spacing between the rods is observed in individual hetero-ZnS-ZnSe nanorod couples (Figures S6-8). The boundaries of the nanorods couples, and in particular the gap between them, became more evident after coating of the hetero-ZnS-ZnSe/ZnS core/shell nanorod couples with a shell layer of CdS (Figure $2 \mathrm{~h}$ ) (see Experimental Section in ESI for details). This is mainly a result of the surface reconstruction of the branched $\mathrm{ZnS}$ structures on the surface of the hetero-nanorod couples, which occurs at the high growth temperature that is required for the CdS shell growth.

High angle annular dark field-scanning transmission electron microscopy (HAADF-STEM) confirms the formation of the branched structures on the surface of hetero-ZnSZnSe/ZnS core/shell nanorod couples (Figure 3a). Some particles that resemble single rods can also be identified in the micrograph, but they exhibit a higher contrast, which indicates that these are rod-couples, which are positioned in a perpendicular alignment on top of amorphous carbon film (Figure 3a). HAADF-EDX element mapping confirms the presence of $\mathrm{Zn}$, Se and $\mathrm{S}$ elements in the hetero-nanorod couples (Figures $3 \mathrm{~b}, \mathrm{~S} 9$ ) and $\mathrm{Zn}^{2+}$ ions are evenly distributed throughout the elongated nanoparticles (Figure $3 \mathrm{~b}$ ). Close inspection on the element maps of $\mathrm{Se}^{2-}$ and $\mathrm{S}^{2-}$ ions indicates $\mathrm{Se}^{2-}$ ions are predominately distributed on the inner side of the obtained nanorod couples whereas $\mathrm{S}^{2-}$ ions are initially located on the outer side of the nanoparticles. This confirms the formation of ZnS-ZnSe/ZnS core/shell nanorod couples accompanied with the extensive growth of ZnS branched structures on the ZnSe rod components. Apparently, the mapping of Se element is weak, which is in consistence with the low molar ratio of Se ( 13\%) in the branched hetero-ZnS$\mathrm{ZnSe/ZnS} \mathrm{core/shell} \mathrm{nanorod} \mathrm{couples} \mathrm{(Figure} \mathrm{1f).} \mathrm{The} \mathrm{Z-}$ contrast STEM image (Figure 3c) shows that the spacing between two rod components is not continuous and may be interrupted by the fuse of the adjacent facets of two rods. This image also confirms that ZnS branched structures have grown on both exterior sides and the interior sides of the rod components of nanorod couples.

One of the intriguing questions regarding the presented system is why does the growth of ZnS on the surface of heteroZnS-ZnSe nanorod couples leads to branched structures. Obviously, this is not due to the compatibility between these two materials since the lattice mismatch between $\mathrm{ZnS}$ (wurtzite) and ZnSe (wurtzite) is not very large (4.5\%) (Table S1). In a control experiment, ZnSe nanorods were dissolved into the same reaction solvent in the presence of the same sulfur precursor, i.e. 1-dodecanethiol, at a reaction temperature of $280{ }^{\circ} \mathrm{C}$. Under these conditions, the ZnSe nanorods were partially etched, leading to rods with rough surface facets (Figure S10). This process highly resembles the etching of CdS nanorods in the presence of zinc oleate. ${ }^{35}$ Thus we believe that in the case of the $\mathrm{ZnS}-\mathrm{ZnSe} / \mathrm{ZnS}$ nanorod couples, the surface of the ZnSe rods is etched, and at the same time, ZnS grows epitaxially on the rough surface facets of the nanorod couples, leading to the formation of branched 

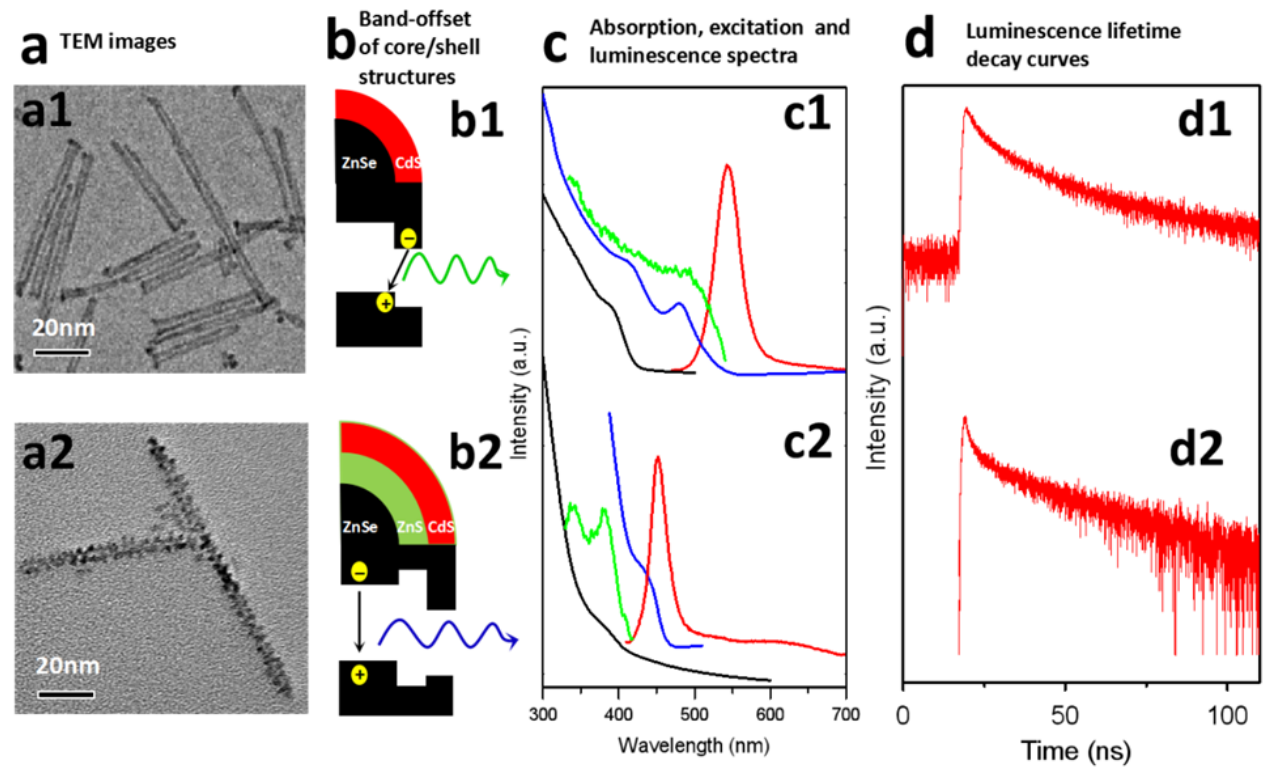

Fig. 5 (a) TEM images. (b) Band-offset of core/shell structures. (c) Optical spectra. (d) Fluorescence lifetime decay curves at room temperature. (a1-d1) Homo- ZnSe nanorod couples. (a2-d2) Branched hetero- ZnS (short arm)-ZnSe (long arm)/ZnCdS core/shell/shell nanorod couples.

\section{structures.}

It is worth noting that the structure of the zinc blende $\mathrm{ZnS}$ short arms connecting the end facets of two parallel ZnSe nanorods is different from that of the wurtzite $\mathrm{ZnS}$ shell grown on the side facets of the ZnSe nanorods. This can be explained by the relatively small difference in the total energy between zinc blende and wurtzite phases of $\mathrm{ZnS}(3.1 \mathrm{meV} / \mathrm{atom})^{36}$ and the different types of growth of ZnS with respect to the preexisting ZnSe. In the former case, the growth of the zinc blende structured ZnS on the wurtzite structured ZnSe nanorods only occurred on a small region, i.e. the most reactive end facets of ZnSe nanorods. This is unlikely lead to substantial lattice strain at the interfaces between the short $\mathrm{ZnS}$ arms and the end facets of ZnSe nanorods. In addition to the effect of lattice mismatch, kinetic effect of reaction precursors may also affect the phase of ZnS because the crystallization of zinc blendestructured $\mathrm{ZnS}$ is preferred at high supersaturation of zinc and sulfur precursors. ${ }^{37-39}$ However, in the latter case, wurtzite structured $\mathrm{ZnS}$ growth on the elongated side facets of wurtzite structured $\mathrm{ZnSe}$ is preferred because this can significant reduce the lattice strain and increase the stability of the core/shell structure.

Electronic structure of hetero-ZnS (short arm)-ZnSe/ZnS core/shell (long arm) nanorod couples

The transition from homo-ZnSe nanorod couples to heteroZnS-ZnSe/ZnS nanorod couples leads to a significant change in the envelope wave functions of the charge carriers and in the energy level structure. A numerical finite-well EMA approach was employed to study the electronic structure of the heteroZnS-ZnSe/ZnS nanorod couples (Figure 4, Table S2, also see ESI for details). In homo-ZnSe nanorod couples, the wave functions of the lowest electron/hole states reside in the corners of the nanorod couples (right panel of Figure 4), while in the hetero- ZnS-ZnSe nanorod couples they reside in the long vertices (Left panel of Figure 4). From the shape and position of the lowest six electronic and hole states, they can be clearly characterized as ZnSe rod states. However, because the electron states energies reside above the conduction band-offset of ZnSe-ZnS (ZB), the penetration of the electron states into the short edges is slightly more pronounced. Within the first 6 states, in the ZnSe there is a relatively large energy difference between the "corner" states and the long edge states, which is a result of the lower confinement of the charge carriers in the corners of the structure. In contrary, in the hetero-ZnS-ZnSe system, all the states are almost degenerate because they are "rod" states. It is worth noting that the formation of the ZnS shell on the hetero- ZnS-ZnSe nanorod couples further strengthens the confinement of holes into the ZnSe nanorod components of the hetero-nanorod couples, while the electrons leak much more into the $\mathrm{ZnS}(\mathrm{W})$ shell, mainly because of the lower effective mass of the electron.

Optical properties of hetero- ZnS-ZnSe/ZnCdS core/shell nanorod couples

The above EMA simulations show that the electronic structures of the hetero- ZnS-ZnSe core/shell nanorod couples should present a distinct change in the luminescence characteristics in comparison to those of the homo-ZnSe nanorod couples (Figure 4). In order to eliminate surface traps and to increase the quantum efficiency, a CdS layer was grown on the nanorod couples, generating hetero-ZnS-ZnSe/ZnCdS core/shell nanorod couples. TEM measurements confirmed the obtained homo-ZnSe/CdS (Figure 5a1) and hetero-ZnS$\mathrm{ZnSe/ZnCdS} \mathrm{core/shell} \mathrm{nanorod} \mathrm{couples} \mathrm{(Figure} \mathrm{5a2)} \mathrm{retain} \mathrm{the}$ distinct features of the original particles (Figures S11a and 12a). Statistical analysis on the nanoparticles before and after 
the CdS shell growth shows that 1-4 monolayers of CdS were grown (Figures S11b and S12b). As depicted in Figure 5b1, ZnSe/CdS nanorod couples constitute core/shell structures with a type II configuration, and therefore, a significant red shift of the band gap with respect to the original ZnSe is expected. The excitation spectra are broader (green curves in Figure $5 \mathrm{c} 1$ ) and resemble the respective absorption spectrum. The corresponding Stokes shift is large $(62 \mathrm{~nm})$ with respect to that of CdS rods ( 4-10 nm, Figure S13), in consistence with previous results reported in the literature. ${ }^{40-42}$

In contrast, the band structure of hetero- $\mathrm{ZnS}-\mathrm{ZnSe} / \mathrm{ZnS}$ core/shell nanorod couples have a quasi-type I configuration because the electron wave functions slightly penetrate the $\mathrm{ZnS}$ while the holes are predominantly confined inside the $\mathrm{ZnSe}$ nanorod components (Figure 5b2), as predicted by the EMA simulations (Figure 4). The formation of an alloyed layer of ZnCdS on hetero ZnS-ZnSe nanorod couples pushes down the band edge of the conduction band slightly (Figure 5b2). As a result, a small red shift on both absorption and emission spectra in comparison with those of homo- ZnSe/CdS core/shell nanorods couples is expected. As for the hetero$\mathrm{ZnS}-\mathrm{ZnSe} / \mathrm{ZnCdS}$ core/shell nanorod couples, interestingly, the luminescence excitation spectrum (green curve in Figure $5 c 2$ ) matches the absorption spectrum of the original hetero- ZnS$\mathrm{ZnSe} / \mathrm{ZnS}$ nanorods couples. The emission of the hetero-ZnS$\mathrm{ZnSe} / \mathrm{ZnCdS}$ core/shell nanorod couples falls in the blue range with a peak at $451 \mathrm{~nm}$ and the Stokes shift is $10 \mathrm{~nm}$. This shift is much smaller than that of homo- $\mathrm{ZnSe} / \mathrm{CdS}$ core/shell nanorod couples with a type II configuration (Figure $5 \mathrm{c} 1$ ) and is consistent with the aforementioned band offset analysis. It is worth noting that the strain existing at the interfaces between ZnS and CdS may also lead to a red shift as observed in core/shell nanoparticles. ${ }^{32}$

We also compared the luminescence spectra of the nanorods couples before and after CdS shell growth (Figure S14). In this case, luminescence of the original nanorods couples was obtained by performing the surface ligand exchange from oleylamine to trioctylphosphine (TOP). After the CdS shell growth, the shifts of the luminescence peak of the homo- ZnS-ZnSe/CdS nanorod couples (Figure S14a) are $120 \mathrm{~nm}$, whereas the shift for hetero- ZnS-ZnSe/ZnCdS core/shell nanorod couples (Figure S14b) is $44 \mathrm{~nm}$. The luminescence spectra for all core/shell nanorods couples measured at $77 \mathrm{~K}$ exhibit a blue shift compared with those measured at room temperature (Figures S15 and S16), which is consistent with the reduction of thermal smearing.

The quantum efficiency of the hetero- $\mathrm{ZnS}-\mathrm{ZnSe} / \mathrm{ZnCdS}$ core/shell nanorod couples is $2 \%$, which is much lower than those of homo- ZnSe/CdS nanorods couples (15\%) (Table S3). This is most likely because forming a perfect CdS shell over the branched ZnS structures in hetero- ZnS-ZnSe/ZnS nanorod couples is hard to achieve, and defects, which act as trap states for the electrons and holes are not diminished. Fluorescence lifetimes of all core/shell nanorod couples at both room temperature and $77 \mathrm{~K}$ and were measured and compared in Figures $5 d$ and S17. The time-resolved fluorescent decay curves were fitted by a bi-exponential model $(t)=$
$A_{1}\left[-t / \tau_{1}\right]+A_{2} \exp \left[-t / \tau_{2}\right]$. For hetero $\mathrm{ZnS}-\mathrm{ZnSe} / \mathrm{ZnCdS}$ nanorod couples, $\tau_{1}=6.0 \mathrm{~ns}$ and $\tau_{2}=45.3 \mathrm{~ns}$ whereas $\tau_{1}=10.3 \mathrm{~ns}$ and $\tau_{2}$ $=50.5 \mathrm{~ns}$ for homo- $\mathrm{ZnSe} / \mathrm{CdS}$ nanorods couples at room temperature (Table S3). The fluorescent lifetimes are much shorter at $77 \mathrm{~K}$ (Figure S17) compared those at room temperature as the possibility for non-radiative transition is significantly reduced at low temperature. Although the heteroZnS-ZnSe/ZnCdS core/shell nanorod couples show a much lower quantum efficiency than homo- $\mathrm{ZnSe} / \mathrm{CdS}$ nanorods couples, the luminescence and luminescence excitation spectra of the former provide sufficient information to reveal the distinct change in the luminescence characteristics between hetero- and homo- nanorod couples.

We further expanded this distinct hetero- nanorod couples into other compositions by a cation exchange reaction (Figure S18a). ${ }^{43-45}$ We specifically targeted lead ions $\left(\mathrm{Pb}^{2+}\right)$ since it is most likely to observe the electronic coupling effect between two closely spaced PbSe rods within a hetero- PbS (short arms)-PbSe (long arms) nanorod couple. This is because the wavefunctions of electrons and holes in $\mathrm{PbSe}$ are more delocalized considering its larger exciton Bohr radii $(46 \mathrm{~nm})^{46}$ in comparison with the small Bohr exciton radius of $\mathrm{ZnSe}(4.5$ $\mathrm{nm}$ ). ${ }^{47}$ TEM (Figure S18b, S18c) and HAADF-STEM (Figure S18d, S18e) imaging confirmed that the produced nanoparticles maintained the original features of the branched heteronanorod couples. STEM-EDX maps confirmed the alternation of the composition of the hetero- nanorod couples (Figure S19). FFT analysis of the selected area in the HRTEM image (Figure S18c) and XRD patterns corroborate the rock-salt structure of the crystal lattice of the obtained nanoparticles (Figure $\mathrm{S} 18 \mathrm{~g}$ ). The absorption features at longer wavelength (2 in Figure S18f) are consistent with the small band gap of PbS$\mathrm{PbSe}$. The hetero- $\mathrm{PbSe}-\mathrm{PbS} / \mathrm{PbS}$ nanorod couples do not show any detectable $\mathrm{PL}$ as the emission intensity in the near-infrared spectral range may be too weak to be detected. The structural analysis and EDX measurement confirm the successful expansion of hetero- PbS-PbSe nanorod couples via a cation exchange reaction, which provides an ideal platform to study the electronic coupling effects between closely spaced nanorods. Further experiments in this area are in progress.

\section{Conclusions}

In conclusion, we have demonstrated a facile wetchemical approach for the synthesis of hetero- ZnS-ZnSe nanorod couples with $\mathrm{ZnS}$ precisely grown on the end facets of two parallelly aligned $\mathrm{ZnSe}$ nanorods. Due to the small contact area between $\mathrm{ZnSe}$ and $\mathrm{ZnS}$ at the apexes of ZnSe nanorods, the growth of zinc blende $\mathrm{ZnS}$ on $\mathrm{ZnSe}$ nanorods may still be epitaxial. The hetero- ZnS-ZnSe/ZnCdS core/shell nanorod couples show a blue emission due to the distinct quantum confinement regime within these unique heterostructures in comparison with the green emission from homo- core/shell nanorod couples. Further expanding the hetero- nanorod couples produced hetero- PbS-PbSe/PbS nanorod couples. The synthetic strategy developed here may provide new insight into the rational design and synthesis of new heterostructures 
with increasing complexity, and the heterostructures obtained here may facilitate the incorporation of such materials into optoelectronic and catalytic applications.

\section{Conflicts of interest}

There are no conflicts to declare.

\section{Acknowledgements}

G. J. conceived the project, designed the experiments and supervised the research. D.C. conducted synthesis, materials characterization and analysis. A.S. performed the EMA simulations. C.S. measured the Z-contrast STEM of the samples and Z.Y. and J.L. analysed the data. H.L., A.W., A.S. and D.C. prepared figures and Supplementary Information sections. L.A.G. and M.M. measured the optical spectroscopy of the materials. A.S. and M.S assisted with HRTEM measurements and structural analysis. G.J. and A.S. wrote the manuscript. All authors discussed the data, mechanisms, and commented on the manuscript. This work was supported by Australian Research Council (ARC) Discovery Early Career Researcher Award (DECRA) (Project ID: DE160100589), and National Natural Science Foundation of China (Grant No. 21701015 and 21811530054). The authors acknowledge the facilities, and the scientific and technical assistance of the Australian Microscopy \& Microanalysis Research Facility at the Centre for Microscopy, Characterisation \& Analysis, The University of Western Australia, a facility funded by the University, State and Commonwealth Governments.

\section{Notes and references}

1 R. Costi, A. E. Saunders and U. Banin, Angew. Chem. Int. Ed., 2010, 49, 4878-4897.

2 G. Jia, Y. Pang, J. Ning, U. Banin and B. Ji, Adv. Mater. 2019, 31, 1900781.

3 K. Wu, J. Chen, J. R. McBride and T. Lian, Science, 2015, 349, 632-635.

4 P. Li, Z. Wei, T. Wu, Q. Peng and Y. Li, J. Am. Chem. Soc., 2011, 133, 5660-5663.

5 R. D. Robinson, B. Sadtler, D. O. Demchenko, C. K. Erdonmez, L.-W. Wang and A. P. Alivisatos, Science, 2007, 317, 355-358.

6 U. Banin, Y. Ben-Shahar and K. Vinokurov, Chem. Mater., 2014, 26, 97-110.

7 A. Vaneski, A. S. Susha, J. Rodríguez-Fernández, M. Berr, F. Jäckel, J. Feldmann and A. L. Rogach, Adv. Funct. Mater., 2011, 21, 1547-1556.

8 Y. Shemesh, J. E. Macdonald, G. Menagen and U. Banin, Angew. Chem. Int. Ed., 2011, 123, 1217-1221.

9 T. Simon, Bouchonville, N.; M. J. Berr, A. Vaneski, A. Adrovic, D. Volbers, R. Wyrwich, M. Döblinger, A. S. Susha, A. L. Rogach, F. Jäckel, J. K. Stolarczyk and J. Feldmann, Nat. Mater., 2014, 13, 1013-1018.

10 S. M. Kim, S. J. Lee, S. H. Kim, S. Kwon, K. J. Yee, H. Song, G. A. Somorjai and J. Y. Park, Nano Lett., 2013, 13, 1352-1358.

11 N. Oh, B. H. Kim, S.-Y. Cho, S. Nam, S. P. Rogers, Y. Jiang, J. C. Flanagan, Y. Zhai, J.-H. Kim, J. Lee, Y. Yu, Y. K. Cho, G. Hur, J. Zhang, P. Trefonas, J. A. Rogers and M. Shim, Science, 2017, 355, 616-619.
12 R. Lavieville, Y. Zhang, A. Casu, A. Genovese, L. Manna, E. Di Fabrizio and R. Krahne, ACS Nano, 2012, 6, 2940-2947.

13 S.-H. Choi, H. B. Na, Y. I. Park, K. An, S. G. Kwon, Y. Jang, M.-H. Park, J. Moon, J. S. Son, I. C. Song, W. K. Moon and T. Hyeon, J. Am. Chem. Soc., 2008, 130, 15573-15580.

14 C. Xu, J. Xie, D. Ho, C. Wang, N. Kohler, E. G. Walsh, J. R. Morgan, Y. E. Chin and S. Sun, Angew. Chem. Int. Ed., 2008, 47, 173-176.

15 C. Xu, B. Wang and S. Sun, J. Am. Chem. Soc., 2009, 131, 4216-4217.

16 J.-S. Choi, Y.-W. Jun, S.-I. Yeon, H. C. Kim, J.-S. Shin and J. Cheon, J. Am. Chem. Soc., 2006, 128, 15982-15983.

17 D. V. Talapin, J. S. Lee, M. V. Kovalenko and E. V. Shevchenko, Chem. Rev., 2010, 110, 389-458.

18 R. E. Schaak and M. E. Williams, ACS Nano, 2012, 6, 84928497.

19 L. Carbone, C. Nobile, M. De Giorgi, F. Della Sala, G. Morello, P. Pompa, M. Hytch, E. Snoeck, A. Fiore, I. R. Franchini, M. Nadasan, A. F. Silvestre, L. Chiodo, S. Kudera, R. Cingolani, R. Krahne and L. Manna, Nano Lett., 2007, 7, 2942-2950.

20 T. Wang, J. Zhuang, J. Lynch, O. Chen, Z. Wang, X. Wang, D. LaMontagne, H. Wu, Z. Wang and Y. C. Cao, Science, 2012, 338, 358-363.

21 Y. Ben-Shahar, F. Scotognella, I. Kriegel, L. Moretti, G. Cerullo, E. Rabani, U. Banin, Nat. Commun., 2016, 7, 10413.

22 N. J. Borys, M. J. Walter, J. Huang, D. V. Talapin and J. M. Lupton, Science, 2010, 330, 1371-1374.

23 J. Dimitrijevic, L. Krapf, C. Wolter, C. Schmidtke, J.-P. Merkl, T. Jochum, A. Kornowski, A. Schüth, A. Gebert, G. Hüttmann, T. Vossmeyer and H. Weller, Nanoscale, 2014, 6, 10413-10422.

24 H. Li, R. Brescia, R. Krahne, G. Bertoni, M. J. P. Alcocer, C. D'Andrea, F. Scotognella, F. Tassone, M. Zanella, M. De Giorgi and L. Manna, ACS Nano, 2012, 6, 1637-1647.

25 G. Jia and U. Banin, J. Am. Chem. Soc., 2014, 136, 1112111127.

26 G. Jia, A. Sitt, G. B. Hitin, I. Hadar, Y. Bekenstein, Y. Amit, I. Popov and U. Banin, Nat. Mater., 2014, 13, 301-307.

27 B. O. Dabbousi, J. Rodriguez Viejo, F. V. Mikulec, J. R. Heine, H. Mattoussi, R. Ober, K. F. Jensen and M. G. Bawendi, J. Phys. Chem. B, 1997, 110, 9463-9475.

28 X. Peng, M. C. Schlamp, A. V. Kadavanich and A. P. Alivisatos, J. Am. Chem. Soc., 1997, 119, 7019-7029.

29 B. Ji, Y. E. Panfil, N. Waiskopf, S. Remennik, I. Popov and U. Banin, Nat. Commun., 2019, 10, 2.

30 M. Lomascolo, A. Cretì, G. Leo, L. Vasanelli and L. Manna, Appl. Phys. Lett., 2003, 82, 418-420.

31 D. Chen, H. Zhang, Y. Li, Y. Pang, Z. Yin, H. Sun, L.-C. Zhang, S. Wang, M. Saunders, E. Barker and G. Jia, Adv. Mater., 2018, 30, 1803351.

32 A. M. Smith, A. M. Mohs and S. Nie, Nat. Nanotechnol., 2009, 4, 56-63.

33 C.-M. Ning, L. Dou and P. Yang, Nat. Rev. Mater., 2017, 2, 17070.

34 T. Mokari and U. Banin, Chem. Mater., 2003, 15, 3955-3960.

35 N. Oh and M. Shim, J. Am. Chem. Soc., 2016, 138, 1044410451.

36 C.-Y. Yeh, Z. W. Lu, S. Froyen and A. Zunger, Phys. Rev. B, 1992, 46, 10085-10097.

37 T. Omata, H. Uesugi and M. Kita, J. Cryst. Growth, 1994, 394, 81-88.

38 P. D. Cozzoli, L. Manna, M. L. Curri, S. Kudera, C. Giannini, M. Striccoli and A. Agostiano, Chem. Mater., 2005, 17, 12961306.

39 W. Chen, A. Karton, T. Hussian, S. Javaid, F. Wang, Y. Pang and G. Jia, CrystEngComm, 2019, DOI: 10.1039/C9CE00078J.

$40 \mathrm{~K}$. Boldt, C. Ramanan, A. Chanaewa, M. Werheid and A. Eychmüller, J. Phys. Chem. Lett., 2015, 6, 2590-2597. 
41 K. Boldt, K. N. Schwarz, N. Kirkwood, T. A. Smith and P. Mulvaney, J. Phys. Chem. C, 2014, 118, 13276-13284.

42 D. Dorfs, A. Salant, I. Popov and U. Banin, Small, 2008, 4, 1319-1323.

43 D. H. Son, S. M. Hughes, Y. Yin and A. P. Alivisatos, Science, 2004, 306, 1009-1012.

44 W. Li, R. Zamani, M. Ibáñez, D. Cadavid, A. Shavel, J. R. Morante, J. Arbiol and A. Cabot, J. Am. Chem. Soc., 2013, 135, 4664-4667.

45 L. De Trizio and L. Manna, Chem. Rev., 2016, 116, 1085210887.

46 H. Du, C. Chen, R. Krishnan, T. D. Krauss, J. M. Harbold, F. W. Wise, M. G. Thomas and J. Silcox, Nano Lett., 2002, 2, 13211324.

47 V. V. Rossin, T. Böttger and F. Henneberger, Phys. Rev. B, 1996, 54, 7682. 